\title{
GAS IN THE NUCLEUS AND DISK OF M31
}

\author{
VERA C. RUBIN* and W. KENT FORD, Jr.* \\ Dept. of Terrestrial Magnetism, Carnegie Institution of Washington
}

\begin{abstract}
Image tube spectra of emission regions in M31 have been studied to determine relative line strengths and abundances. Lines of $\mathrm{H}, \mathrm{He},[\mathrm{NII}],[\mathrm{SII}],[\mathrm{OI}],[\mathrm{OII}]$, [O III] are observed. The analysis indicates: (1) that there are ions whose line strengths, relative to $\mathrm{H} \alpha$, are a function of the position of the emission region in M31; (2) abundance differences or different excitation mechanisms exist in regions separated by only a few hundred parsecs.
\end{abstract}

\section{Introduction}

For several years we have been obtaining image tube spectra of emission regions in M31. The spectra are centered at $\mathrm{H} \alpha$, and include the Balmer lines $\mathbf{H} \beta, \mathbf{H} \gamma, \mathbf{H} \delta$; [S $\mathrm{SI}] \lambda \lambda 6717,6731 ;$ [N $\mathrm{NI}] \lambda \lambda 6548,6583 ;$ [O III] $\lambda \lambda$ 5007, 4959; [OHI] $\lambda 3727$; [OI] $\lambda 6300$; HeI $\lambda$ 5876. On a few well exposed spectra, lines of [AIV] $\lambda$ 7238, [AII] $\lambda$ 7136, HeI $\lambda$ 6678, [NII] $\lambda$ 5755, and [SHI $\lambda 4071$ are also seen. From the observed line strengths, it is now possible to answer the following questions. (1) Are there ions whose line strengths, relative to hydrogen, are a function of the position of the emission region in the galaxy? The answer is yes. (2) Is it possible to explain the observed line ratios by a single $H_{\text {II }}$ region chemical abundance, with only changes in $T_{e}, N_{e}$, and ionization from region to region? The answer is no: abundance differences or different excitation mechanisms exist in regions separated by only a few hundred parsecs. We now indicate how these results are obtained.

\section{Relative Line Strengths}

Line intensities, relative to $I(\mathrm{H} \alpha)$, have been determined for about 50 regions; for half of these we have two or more plates. Details of the photometry and a catalog of line intensities will be published elsewhere. All intensities are corrected for extinction and spectral response. From the duplicate values, we estimate that the mean error of the line ratio for a strong or moderate line is less than $20 \%$; for a weak line the error may be as great as $30 \%$. Because the ratios $I([\mathrm{OIII}]) / I([\mathrm{OII}]) / I([\mathrm{OI}])$ cannot be reconciled with collisional excitation mechanisms, we must assume that the excitation is radiative (Peimbert, 1968). Then the $I(\mathrm{H} \alpha) / I(\mathrm{H} \beta)$ ratio is predicted to be 2.84 .

From the observed $I(\mathrm{H} \alpha) / I(\mathrm{H} \beta)$ ratio for each region, the logarithmic reddening correction, $c$, has been computed, adopting the reddening function tabulated by Seaton (1960). Values of $c$ range from 0.1 to 2.0. Near the nucleus, there is a large spread in values, with $\bar{c}=1.0$. Near $R=24 \mathrm{kpc}$, the spread has decreased, and $\bar{c}=0.5$.

* Visiting Astronomer, 1966, 1967, 1968, 1969 Lowell Observatory, and Visiting Astronomer 1967, 1968, 1969 Kitt Peak National Observatory, operated by the Association of Universities for Research in Astronomy, Inc. under contract with the National Science Foundation. 
Except for the general decrease in reddening with increasing distance from the nucleus, there is no marked correlation of reddening with position of the spiral arms in M31. For the stars in M31, a value of $c=0.1$ is typical; hence, most of the reddening observed here arises in the emission regions. For emission regions in our Galaxy, Orion, M8, and M17, values of $c$ (Peimbert and Costero, 1969) cover the range 0.38 to 1.7. All line strengths discussed below are corrected for reddening, although the ratios are generally insensitive to this correction because the wavelength region considered is usually small.

The intensity ratio $I([\mathrm{NII}] \lambda 6583) / I(\mathrm{H} \alpha)$ is greater than unity only for emission regions near the nucleus and decreases to $<0.2$ for $R$ near $22 \mathrm{kpc}$. Values of this ratio

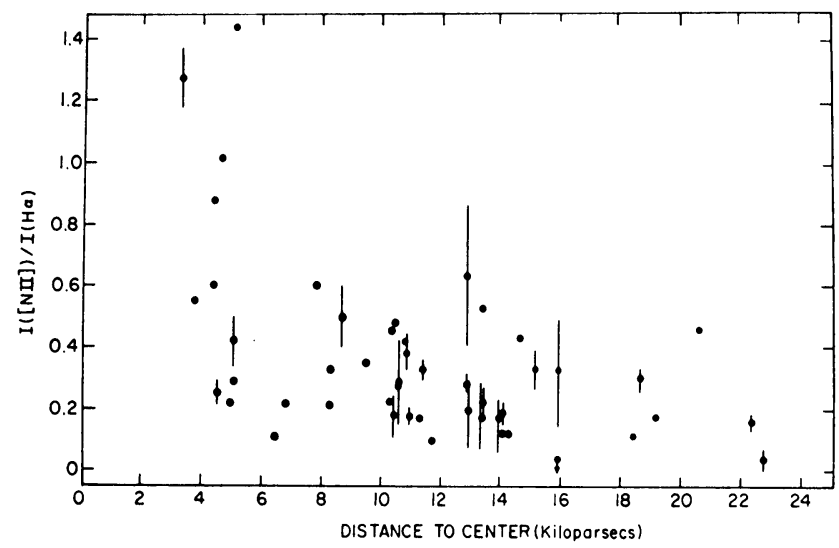

Fig. 1. Intensity ratio $I([\mathrm{NII}] \lambda 6583) / I(\mathrm{H} \alpha)$ for emission regions in $\mathrm{M} 31$, as a function of distance from the center. Error bars indicate the total range of values from two or more plates.

are plotted in Figure 1. From the integrated spectra of galaxies, it is known that $I([\mathrm{~N} I \mathrm{I}])>I(\mathrm{H} \alpha)$ in the nuclei of some galaxies. This effect is seen here for individual emission regions as well.

The ratio $I([\mathrm{~S} I \mathrm{II}] \lambda 6717+\lambda 6731) / I(\mathrm{H} \alpha)$ exhibits an equally striking variation with $R$. For emission regions near the nucleus, this ratio is as large as 2 , but for $R>5 \mathrm{kpc}$ it is generally near 0.1 . In all cases with $I([\mathrm{NII}])>I(\mathrm{H} \alpha)$, then $I([\mathrm{SII}])>I(\mathrm{H} \alpha)$ also. Even for regions with $I([\mathrm{SII}])$ and $I([\mathrm{NII}])<I(\mathrm{H} \alpha)$, there is a correlation of $I\left(\left[\mathrm{~N}_{I I}\right]\right)$ with $I([\mathrm{SH}])$.

The $[\mathrm{OIII}] / \mathrm{H} \beta$ intensity ratios indicate Aller excitation classes from 2 to 4, with the regions of higher excitation generally at greater distances from the nucleus. The principal exceptions to this relation are some regions close to the nuclear bulge $(R=4$ or $5 \mathrm{kpc}$ ), which exhibit high [OIII]/H $\beta$ ratios.

The observed values of the helium to hydrogen ratio, $I(\mathrm{He} \lambda 5876) / I(\mathrm{H} \alpha)$, show a large scatter at all $R$, with values from 0.01 to almost 0.1 . For 21 regions, no He $\lambda 5876$ line is seen, indicating an upper limit of 0.03 to this ratio. There is no indication of an increase of the helium/hydrogen ratio with decreasing $R$.

Perhaps more significant than these variations in the relative line strengths with 
distance from the nucleus is the fact that the 50 spectra can be placed into 8 classes, with similar strengths of [OI], [OII], [O III], [N $\mathrm{NI}$, and [S $\mathrm{SI}$, relative to $\mathrm{H} \alpha$, within each class. In what follows we shall discuss two of these spectral groups.

We show in Table I the relative line strengths for 10 emission regions, identified by Baade and Arp (1964) as the emission regions closest to the nucleus of M31. These spectra fit into two very different groups on the basis of relative line strengths.

TABLE I

Relative line ratios for $\mathrm{HII}$ regions near nucleus of $\mathrm{M} 31$.

\begin{tabular}{cllllll}
\hline $\begin{array}{l}\text { Region } \\
\text { Arp No. }\end{array}$ & $\begin{array}{l}R \\
(\mathrm{kpc})\end{array}$ & $\frac{I([\mathrm{SH}])}{I(\mathrm{H} \alpha)}$ & $\frac{I([\mathrm{NII}])}{I(\mathrm{H} \alpha)}$ & $\frac{I([\mathrm{OII}])}{I(\mathrm{H} \beta)}$ & $\frac{I([\mathrm{OII}])}{I(\mathrm{H} \alpha)}$ & $c$ \\
\hline 55 & 3.2 & 1.34 & 1.69 & 3.1 & $\geqslant 1$ & 0.40 \\
415 & 3.7 & 1.74 & 0.73 & - & - & - \\
521 & 4.4 & 0.76 & 1.17 & 2.4 & $>1$ & 0.66 \\
23 & 4.6 & 1.00 & 1.35 & 1.5 & $=1$ & 0.74 \\
416 & 5.1 & 1.68 & 1.92 & 4.4 & & 0.78 \\
$521 \mathrm{a}$ & 4.4 & $<0.12$ & 0.80 & - & -1 & - \\
519 & 4.5 & 0.14 & 0.32 & 0.5 & $<1$ & 1.2 \\
74 & 4.9 & 0.05 & 0.29 & 0.5 & $<1$ & 1.1 \\
75 & 5.0 & 0.06 & 0.59 & 0.2 & $<1$ & 1.1 \\
423 & 5.1 & 0.29 & 0.56 & 0.2 & &
\end{tabular}

[SII]: $\lambda 6717+\lambda 6731$

$[\mathrm{NII}]: \lambda 6548+\lambda 6583$

[OIII]: $\lambda 5007$

[OII]: $\lambda 3727$

For the strong line group, the intensities of the forbidden sulphur doublet and the forbidden nitrogen doublet are greater than the $\mathrm{H} \alpha$ intensity; [O III], [OII] and [OI] are also strong. It is unlikely that this is an ionization effect; where [OIII] is strong, [SII] is generally weak, for its ionization potential is low, so most [SII] would be ionized to [SIII]. In the second group, (the normal line group) the sulphur intensity is only one-tenth that of $\mathrm{H} \alpha$; all other ions are relatively weaker also.

On Baade's 100-in. plates, there are no significant differences between the appearances of these 10 emission regions, nor are they separated spatially into two groups. Regions 521 (strong line) and 521a (normal line) are located within a hundred parsecs of each other, and spectra of both objects are contained on a single plate. Their radial velocities are in no way peculiar, although the velocity dispersion near $R=4 \mathrm{kpc}$ is the largest observed. (Rubin and Ford, 1970.) Curiously, the reddening coefficient, $c$, is significantly different in the two types of regions; for the strong line group, $\bar{c}=0.64$, while for the normal line group, $\bar{c}=1.2$. This difference must indicate either different properties of the interstellar medium at the positions of the two types of regions, or different excitation mechanisms, producing an $\mathrm{H} \alpha / \mathrm{H} \beta$ ratio different from that predicted by radiative excitation.

We show in Figure 2 the line strengths, relative to $\mathrm{H} \alpha$, for all 8 spectral groups. For each group, the mean distance to the nucleus, $\bar{R}$, and the number of emission 
regions in the group, $n$, are indicated. For $R \geqslant 5 \mathrm{kpc}$, all lines are weaker than $\mathrm{H} \alpha$, [NII] generally decreases with $R$, and [S $\mathrm{SI}$ is generally near 0.1 , except for a group of 10 regions centered near $12 \mathrm{kpc}$, in which $[\mathrm{SII}] / \mathrm{H} \alpha$ is near 0.3 . For comparison, corresponding line strengths for Orion, M8, M17 (Peimbert and Costero, 1969) are included. About half of the emission regions in $M 31, R \Im 8 \mathrm{kpc}$, are 'Orion-like', while the remaining regions have $[\mathrm{NII}]$ and $[\mathrm{SII}]$ lines which are about twice as intense, relative to $\mathrm{H} \alpha$, as the corresponding lines in Orion.

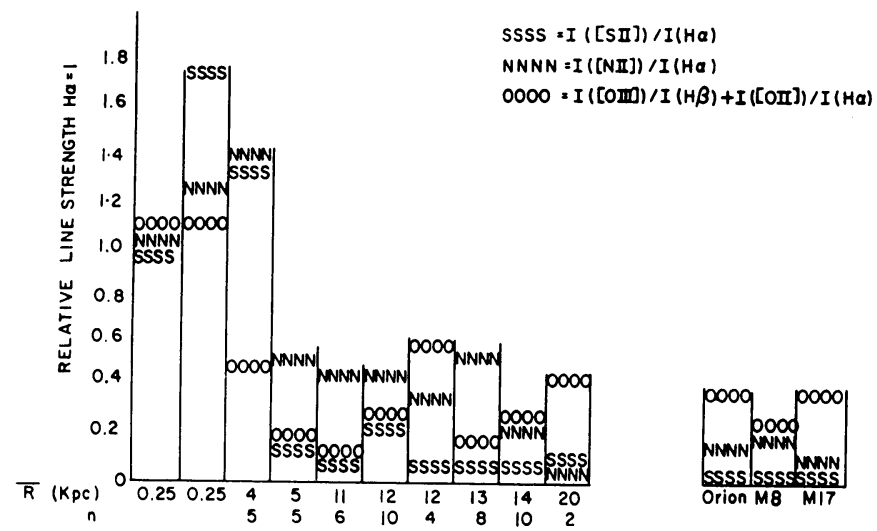

Fig. 2. Line strengths, relative to $\mathrm{H} \alpha$, for emission regions in M31. Regions have been grouped by spectral properties. The mean distance to the center $R$, and the number of regions in each group, $n$, are shown. For $R=0.25 \mathrm{kpc}$, line intensities come from spectra taken across the nuclear bulge.

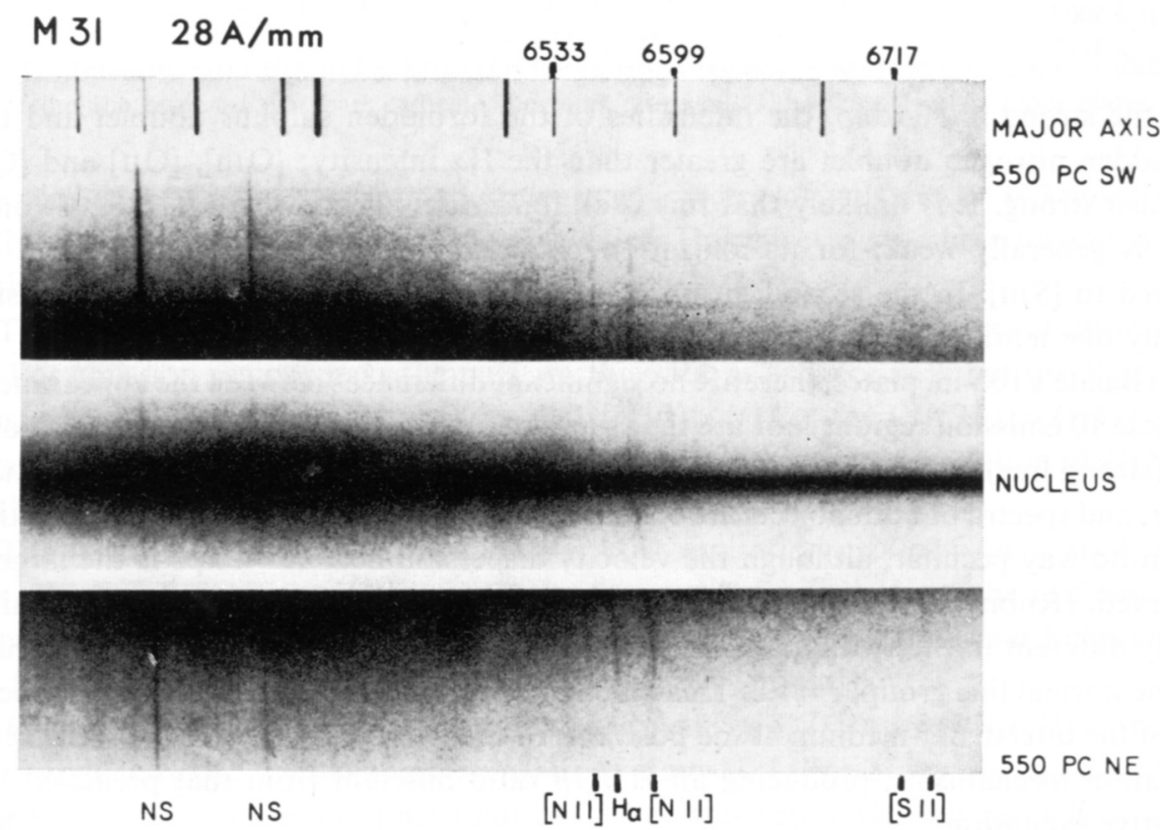

Fig. 3. Image tube spectra across the nuclear bulge in $M 31$, dispersion $28 \AA / \mathrm{mm}$, showing emission lines of $[\mathrm{NII}] \lambda 6548, \mathrm{H} \alpha,[\mathrm{NII}] \lambda 6583$, and [S $\mathrm{II}] \lambda \lambda 6717$ and 6731 . Slit along major axis. Exposure times $5^{\mathrm{h}} 43^{\mathrm{m}}, 2^{\mathrm{h}}, 5^{\mathrm{h}} 46^{\mathrm{m}}$, with 84 -in. Kitt Peak reflector. 
For $R<3 \mathrm{kpc}$, no emission regions are identified in M31. However, high dispersion spectra across the nuclear bulge show emission lines of $[\mathrm{NII}] \lambda 6548, \mathrm{H} \alpha,[\mathrm{NII}] \lambda 6583$, [SII] $\lambda \lambda 6717$ and 6731 , and [O III] $\lambda$ 5007. The intensity ratio $I([\mathrm{NII}] \lambda 6583) / I(\mathrm{H} \alpha)$ is generally about 2 , although the ratio changes to 1 in some regions. Abrupt changes in the line ratios occur in dust lanes across the nuclear bulge. We show in Figure 3 three spectra taken along the major axis of M31, showing the emission lines in the red spectral region. Relative line intensities, measured at $R=0.25 \mathrm{kpc} \mathrm{SW}$ and $0.25 \mathrm{kpc} \mathrm{NE}$ are plotted in the first two columns of Figure 2. Just as in the strong line group of emission regions near the nucleus, $[\mathrm{SII}],[\mathrm{NII}],[\mathrm{OIII}]$, and $\left[\mathrm{O}_{\mathrm{II}}\right]$ are strong in the nuclear regions.

\section{Relative Abundances}

We will discuss only very generally the transformation from relative line strengths to relative abundances. If we assume radiative excitation, then the absence of the [NII] $\lambda 5755$ line implies $T_{e}<11000^{\circ}$ in general, while the ratio of the [SII] doublet implies $N_{e} \leqslant 10^{4}$, except in the nucleus where high densities exist. The ratio of total chemical abundances of oxygen to hydrogen, $\mathrm{N}(\mathrm{O}) / \mathrm{N}(\mathrm{H})$, is then given by $\left(\mathrm{N}\left(\mathrm{O}^{+}\right)+\mathrm{N}\left(\mathrm{O}^{++}\right)\right) / \mathrm{N}\left(\mathrm{H}^{+}\right)$, where the ratios of the ions are obtained from expressions given by Peimbert and Costero (1969). The procedure is similar for $N(S) / N(H)$. For $\mathrm{N}^{++}$, which is not observed, and $\mathrm{S}^{++}$, which is not observed, we use ratios of ions of oxygen with similar ionization potentials.

For the strong line group of emission regions near the nucleus of $\mathrm{M} 31$, no choice of $T_{e}$ can reproduce the solar abundance for $\mathrm{O}, \mathrm{N}$, and $\mathrm{S}$. If $\mathrm{N}(\mathrm{O}) / \mathrm{N}(\mathrm{H})$ is chosen to be solar, then $T_{e} \sim 8000^{\circ}$, nitrogen is overabundant by a factor of about 2 , and sulphur is overabundant by a factor of about 25 . Even for a high $T_{e}$, sulphur is still overabundant, and oxygen then becomes underabundant.

For the normal line group, nitrogen and oxygen have the solar abundance for $T_{e}=7000^{\circ}$, and sulphur is overabundant by a factor of 2 , values which are like those for emission regions in our Galaxy (Peimbert and Costero, 1969). The remaining emission regions in M31 have relative abundances which do not vary by more than factors of 2 or 3 from those in galactic emission regions. Details for all regions will be published elsewhere.

The mean helium abundance, for 15 regions with measurable $\mathrm{HeI} \lambda 5876$ lines, is $\mathrm{N}(\mathrm{He}) / \mathrm{N}(\mathrm{H} \alpha)=0.12$. However, if we include 21 additional regions with low values for the upper limit to this line intensity, then $\mathrm{N}(\mathrm{He}) / \mathrm{N}(\mathrm{H} \alpha)<0.08$. The He II $\lambda 4686$ line is never observed: this implies $\mathrm{N}\left(\mathrm{He}^{++}\right) / \mathrm{N}\left(\mathrm{H}^{+}\right)<5 \times 10^{-4}$.

\section{Conclusions}

If the observed line ratios in M31 are transformed to relative chemical abundances, using conventional radiative excitation theory, then the abundance of sulphur become abnormally high in the nuclear bulge and in some emission regions near the nucleus. The remaining emission regions have abundances which differ only by small factors 
(2-3) from galactic emission regions. Hence it appears that chemical abundance differences must exist in regions separated by only hundreds of parsecs, or excitation mechanisms must differ over the same distances. From the present observations, it is not possible to choose between these two possibilities, although no simple mechanism that can enhance the sulphur lines is apparent. The low $\mathrm{H} \alpha / \mathrm{H} \beta$ ratio observed for the strong line regions makes it unlikely that the excitation is collisional, unless $T_{e}$ is very high $\left(\sim 40000^{\circ}\right)$. At this $T_{e}$, the intense sulphur line would not be predicted, so models with various regions and various $T_{e}$ would have to be invoked.

In our Galaxy, emission regions with abnormally strong sulphur lines are unknown, although observations are scarce, and do not extend to regions near the nucleus. Only the Crab nebula (Minkowski, 1942; Woltjer, 1958) and supernova remnants (Parker, 1964) have [S II] lines stronger than H $\alpha$. In M31, a single supernova remnant would be difficult to observe. If many supernovae recently enriched the interstellar medium in some regions near the nucleus of M31, the helium abundance would be expected to be higher than is observed. It is possible that the mechanism enhancing the sulphur lines in supernova remnants is also operating near the nucleus of M31. For the galactic supernova remnants, it is generally concluded that the [ $\left.\mathrm{S}_{\mathrm{II}}\right]$ originates in a zone of lower temperature than do the lines of higher ionization.

Among our collection of spectra of nuclei of external galaxies, only for two, M51 and NGC 1052, is the intensity of the sulphur doublet $(\lambda 6717+\lambda 6731)$ greater than the $\mathrm{H} \alpha$ intensity. For both these nuclei also, $I([\mathrm{NII}])>I(\mathrm{H} \alpha)$. These spectra are reproduced in Figure 4 and values of the line strengths, corrected for extinction,

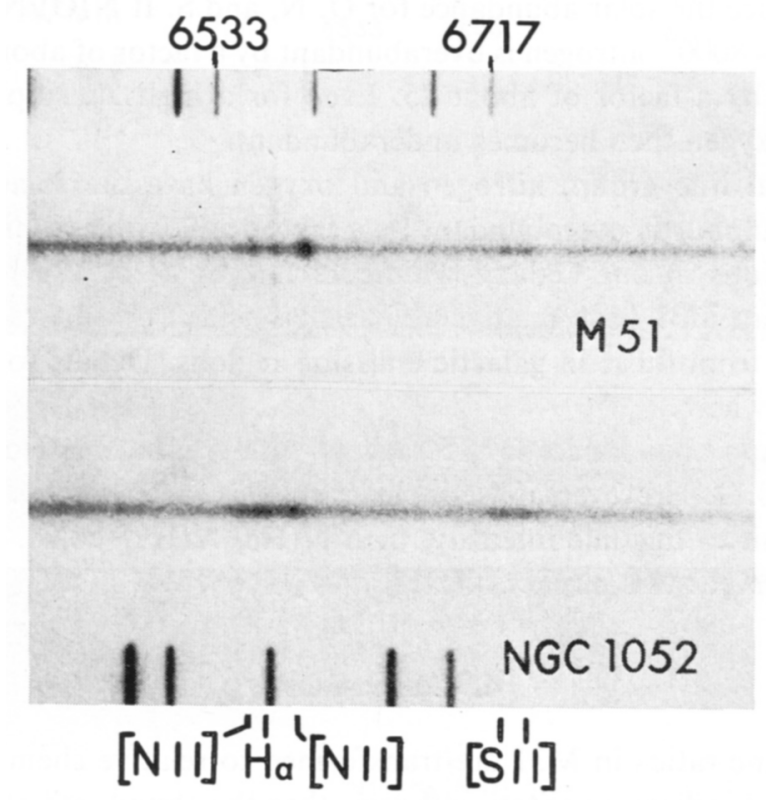

Fig. 4. Image tube spectra of M51 and NGC 1052̇, dispersion $66 \AA / \mathrm{mm}$. Exposure times $38^{\mathrm{m}}, 48^{\mathrm{m}}$ at the Perkins 72-in. telescope. For M51, emission extends 75", slit EW. Spectrum of NGC 1052 (larger redshift) has been displaced so emission features are in line with those in M51. 
TABLE II

Emission line strengths for nuclei of M51, NGC 1052, and M81; H $\alpha=1.0$

\begin{tabular}{lllll}
\cline { 5 - 5 } Nucleus & {$\left[\mathrm{N}_{\mathrm{II}}\right] \lambda 6583$} & {$[\mathrm{SII}] \lambda 6717$} & {$[\mathrm{SII}] \lambda 6731$} & {$[\mathrm{~S}$ II] $] \lambda 6717+\lambda 6731$} \\
\hline M51 & 3.8 & 0.95 & 0.98 & 1.9 \\
NGC 1052 & 1.1 & 0.82 & 0.68 & 1.5 \\
M81 & 1.6 & 0.14 & 0.15 & 0.3
\end{tabular}

reddening and spectral response, are listed in Table II. In M81, the forbidden nitrogen lines are stronger than $\mathrm{H} \alpha$, but $I([\mathrm{SII}]) / I(\mathrm{H} \alpha)=0.3$. For each galaxy, we have only a single calibrated plate, so the values may be revised later. In no other galaxies have we observed [SII] lines as great as one-tenth of the $\mathrm{H} \alpha$ intensity. More observations and studies of the [SII] doublet are desirable, because an understanding of its peculiar strength in the nuclei of some galaxies and some emission regions would add to our small store of knowledge concerning galactic nuclei.

\section{Acknowledgements}

We wish to thank Dr John Hall, Director of the Lowell Observatory, and Dr N. U. Mayall, Director of the Kitt Peak National Observatory, for making telescope time available.

\section{References}

Baade, W.: 1963, Evolution of Stars and Galaxies, Harvard University Press, Cambridge, p. 59.

Baade, W. and Arp, H. C.: 1964, Astrophys. J. 139, 1027.

Minkowski, R.: 1942, Astrophys. J. 96, 199.

Parker, R. A. R.: 1964, Astrophys. J. 139, 493.

Peimbert, M.: 1968, Astrophys. J. 154, 33.

Peimbert, M. and Costero, R.: 1969, Bol. Obs. Tonantzintla Tacubaya 5, 3.

Rubin, V. C. and Ford, W. K., Jr.: 1970, Astrophys. J. 159, 379.

Seaton, M. J.: 1960, Rep. Prog. Phys. 23, 313.

Woltjer, L.: 1958, Bull. Astron. Inst. Neth. 14, 39.

\section{Discussion}

King: Will the velocity fields be measured and published?

Mrs. Rubin: Velocities of the HII regions are published. (Rubin and Ford: 1970, Astrophys. J. 159, 379.) Velocities of the excited gas in 16 position angles across the nuclear bulge have been measured but are not yet understood. 